\title{
ALCOHOL AND CARDIOVASCULAR DISEASE: POSITION PAPER OF THE CZECH SOCIETY OF CARDIOLOGY
}

\author{
Renata Cífková1, 2 , Alena Krajčoviechová1 \\ ${ }^{1}$ Centre for Cardiovascular Prevention, First Faculty of Medicine, Charles University and Thomayer Hospital, Prague, Czech Republic \\ ${ }^{2}$ Department of Medicine II, First Faculty of Medicine, Charles University, Prague, Czech Republic
}

\begin{abstract}
SUMMARY
Epidemiologic studies consistently report a U-shaped curve relationship between the amount of alcohol consumption and cardiovascular disease, with consumption of $\geq$ three alcoholic drinks being associated with an increased risk. However, the cardioprotective effect of light and moderate alcohol consumption has been recently questioned. In the absence of a randomized trial confirming the cardioprotective effect of light or moderate alcohol consumption, an alternative method to prove the causality is Mendelian randomization using a genetic variant serving as a proxy for alcohol consumption. A Mendelian randomization analysis by Holmes et al. suggests that a reduction in alcohol intake is beneficial for cardiovascular health also in light to moderate drinkers. In a recent analysis of 83 prospective studies, alcohol consumption was roughly linearly associated with a higher risk of stroke, coronary heart disease excluding myocardial infarction, heart failure and risk of death from aortic aneurysm dissection. By contrast, increased alcohol consumption was associated with a lower risk of myocardial infarction. "Low-risk" alcohol consumption recommended by the National Institute of Public Health, Czech Republic, should not exceed $16 \mathrm{~g}$ of $100 \%$ ethanol/day for women and $24 \mathrm{~g} / \mathrm{day}$ for men; at least two days a week should be alcohol free, and the dose of ethanol during binge drinking should not exceed $40 \mathrm{~g}$. In practice, this means one standard drink daily for five days at most and two standard drinks at most when binge drinking. These amounts should be considered the highest acceptable limits, but alcohol consumption in general should be discouraged.
\end{abstract}

Key words: cardioprotective effect of alcohol, alcohol consumption, Mendelian randomization, heavy drinking, moderate drinking, light drinking

Address for correspondence: R. Cífková, Centre for Cardiovascular Prevention, Charles University in Prague, First Faculty of Medicine and Thomayer Hospital, Vídeňská 800, 14059 Prague 4, Czech Republic. E-mail: renata.cifkova@ftn.cz

https://doi.org/10.21101/cejph.a5998

\section{INTRODUCTION}

Epidemiologic studies consistently report a U-shaped curve relationship between the amount of alcohol consumed and cardiovascular disease (CVD). While consumption of three or more alcoholic drinks per day is associated with an increased risk of developing CVD (1), light alcohol consumption (one to two drinks per day) appears to be associated with a lower risk of developing coronary heart disease (CHD) and stroke compared with no alcohol consumption. The lowest risk was found to be associated with consumption of approx. 12-25 British units of alcohol per week ( 1 British unit of alcohol $=10 \mathrm{ml}$ ethanol $=7.9 \mathrm{~g}$ ethanol $)$ (2). However, the cardioprotective effect of light and moderate alcohol consumption has been recently questioned. First, in epidemiologic studies, confounding effects of lifestyle and social factors cannot be excluded. Second, the cardioprotective effect of light to moderate alcohol consumption can be explained by a pre-existing increased cardiovascular risk due to poor health of non-drinkers (3). Finally, the mechanism of the cardioprotective effect of moderate alcohol consumption is likewise not fully explained from a pathophysiological point of view. The most often proposed explanation is an increase in HDL-cholesterol levels. However, whether the association of increased HDL cholesterol with decreased risk of developing CVD is causal remains unclear (4). Also, while an increase in HDL cholesterol has been reported in interventional alcohol studies, the small sample size and short follow-up question the validity of their results.

\section{Mendelian Randomization}

In the absence of a randomized trial confirming or refuting a causative relationship between light or moderate alcohol consumption and decreased incidence of CVD, an alternative method is to use a genetic variant that serves as a proxy for alcohol consumption; this approach is called Mendelian randomization (5).

Observational studies employing Mendelian randomization are currently considered the gold standard in assessing the causative role of a biomarker in the development of a disease. Compared with conventional observational studies, the advantage of the former is that the incidence of the genetic variant used in an individual is unaffected by other confounding external factors and reverse causality. Studies using Mendelian randomization in cardiovascular epidemiology have confirmed the relevance of novel biomarkers, e.g. lipoprotein(a), and have successfully identified therapeutic targets for novel drug classes, e.g. proprotein convertase subtilisin/kexin type 9 (PCSK9) inhibitors. Nonethe- 
less, Mendelian randomization can also be used in cases where the marker being tested is an external factor (alcohol consumption) (5).

The prerequisites for a study employing Mendelian randomization include:

- The genetic variant is closely related to the marker (alcohol consumption in our case).

- The genetic variant is not associated with other confounding external factors.

- The genetic variant is related to the disease only through the marker being tested (thus excluding, in other words, the pleiotropic effect of the genetic variant).

Alcohol dehydrogenase is an enzyme playing a crucial role in alcohol metabolism; it exists in several forms encoded by various genes (6). Members of this enzyme family metabolize a variety of substrates including ethanol, retinol, other aliphatic alcohols, hydroxysteroids and lipid peroxidation products. The non-synonymous single-nucleotide polymorphism (SNP) rs1229984 (guanine to adenine) in the alcohol dehydrogenase 1B
(ADH1B) gene leads to an amino-acid substitution of arginine for histidine at position 47. Carriers of the ADH1B rs1229984 A allele respond to alcohol consumption by flushing, they consume less alcohol and have lower blood ethanol levels, and are at lower risk of developing addiction to alcohol in adults and adolescents.

A meta-analysis of Holmes et al. (7) reported results of an international collaborative project using the ADH1B rs1229984 A allele as a tool to investigate the relationship between alcohol consumption and cardiovascular events in over 260,000 individuals. The project analyzed data from 56 studies with a total of 261,991 participants of European descent; 48\% were women whose mean age was 58 years. Overall, 20,259 coronary events, 10,164 strokes and 14,549 cases of type 2 diabetes were recorded.

\section{ADH1B and Alcohol Consumption}

The ADH1B rs1229984 A allele has proved to be a suitable surrogate marker of alcohol consumption. Carriers of the rs 1229984

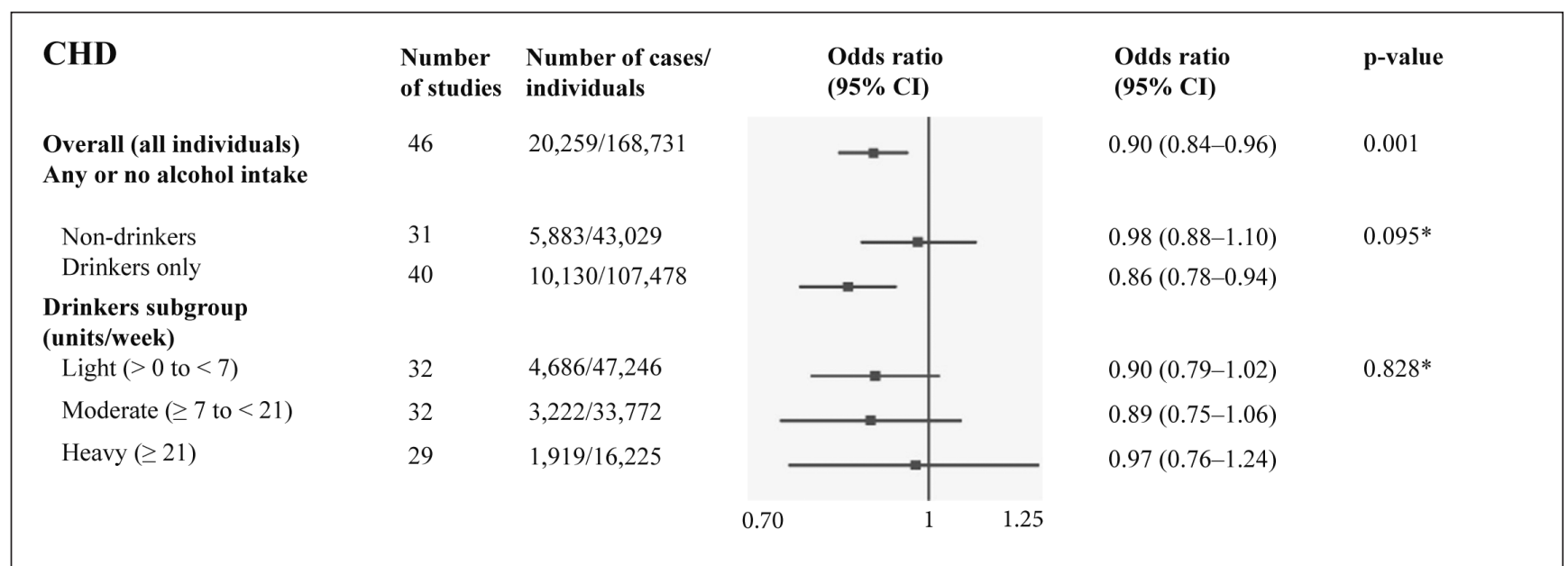

Fig. 1. Meta-analysis pooled estimates of the association between ADH1B rs/229984 (A-allele carriers versus non-carriers) and coronary heart disease overall, and stratified by alcohol intake. Adapted from Holmes MV et al. (7).

$\mathrm{CHD}$ - coronary heart disease; $\mathrm{Cl}$ - confidence interval

${ }^{*} \mathrm{p}$-value for heterogeneity obtained from test for trend using meta-regression

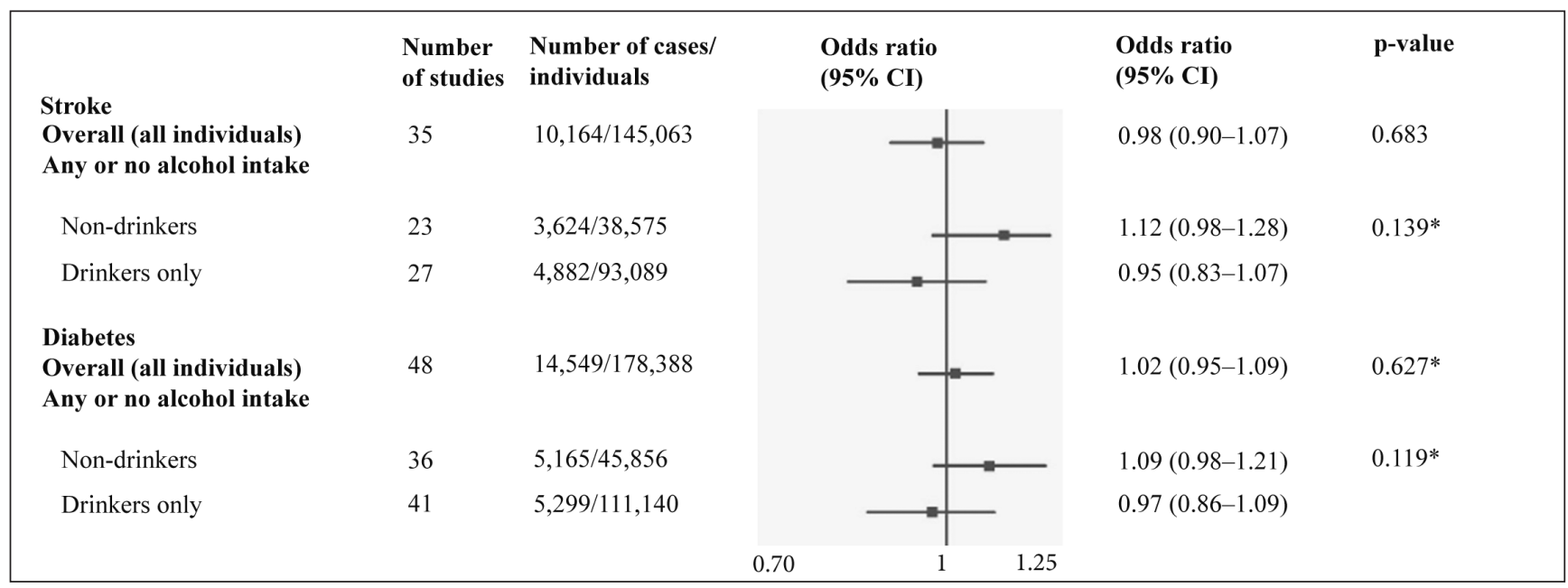

Fig. 2. Meta-analysis pooled estimates of the association between ADH1B rs/229984 (A-allele carriers versus non-carriers) and stroke (combined subtypes) and type-2 diabetes overall, and stratified by alcohol intake. Adapted from Holmes MV et al. (7). 
A allele consumed fewer units of alcohol per week and were at lower risk of falling into the tertile consuming the highest volume of alcohol. Likewise, they were at a lower risk of "binge drinking" (i.e., single excessive alcohol consumption, usually at least $50 \mathrm{~g}$ in men), tended to be self-reported abstainers and showed lower gamma-glutamyl transferase levels.

\section{ADH1B and Cardiovascular Biomarkers}

Carriers of the ADH1B rs1229984 A allele had lower systolic blood pressure, were less likely to develop hypertension, had lower levels of interleukin 6 and C-reactive protein, a lower body mass index and a smaller abdominal circumference. Besides, they were found to have lower levels of non-HDL cholesterol.

However, comparable HDL cholesterol levels were found in both carriers and non-carriers of the ADH1B rs1229984 A allele.

This association probably cannot be explained by the special characteristics of non-drinkers, although the possibility of residual bias and reverse causality cannot be completely ruled out.

\section{ADH1B and Cardiovascular Events}

Carriers of the ADH1B rs1229984 A allele were at lower risk of developing CHD (OR 0.90; 95\% CI 0.84-0.96) and ischaemic stroke (OR 0.83; 95\% CI 0.72-0.95) (Figures 1 and 2).

This study (7) confirms the causative role of alcohol in the aetiology of CVD. Unlike epidemiologic studies published to date, carriers of the ADH1B rs1229984 A allele and, hence, individuals genetically predisposed to lower alcohol consumption and, consequently, to non-drinking, had a more favourable cardiovascular profile and decreased incidence and prevalence of CHD and ischaemic stroke compared with individuals without this genetic variant. Contrariwise, no association between the rs1229984 A allele and levels of HDL cholesterol, physical activity, number of cigarettes smoked per day, packs of cigarettes smoked per year and cotinine levels was observed.

To sum up, the meta-analysis of Holmes et al. (7) suggests that a reduction in alcohol intake is beneficial for cardiovascular health also in light to moderate drinkers. Nevertheless, the results of the above study should be interpreted with caution as, in general, a pleiotropic effect of the studied genetic variant as well as interaction between the gene and external factors cannot be completely ruled out.

\section{Amount of Alcohol Consumption and Cardiovascular Mortality}

The recommended amount of alcohol consumption deemed to be associated with a low risk of total and cardiovascular mortality varies substantially among countries (Table 1). A paper published recently in the Lancet (8) reported data of 599,912 alcohol drinkers obtained from 83 prospective studies conducted in high-income countries and documenting overall 40,310 deaths and 39,018 cardiovascular events (5.4 million person-years of follow-up).

The lowest risk of overall mortality was found in individuals consuming approximately 100 or fewer grams of alcohol per week. Alcohol consumption was roughly linearly associated with a higher risk of stroke (HR 1.14; 95\% CI 1.10-1.17), CHD excluding myocardial infarction (HR 1.06; 95\% CI 1.00-1.11), heart failure (HR 1.09; 95\% CI 1.03-1.15) and risk of death from aortic aneurysm dissection (HR 1.15; 95\% CI 1.03-1.28). By contrast, increased alcohol consumption was associated with a lower risk of myocardial infarction (HR 0.94; 95\% CI 0.91-0.97).

Results of this study support limits for alcohol consumption that are lower than those recommended by the majority of current guidelines.

\section{Recommendations for Consumption of Alcoholic Beverages}

In 2015, the National Institute of Public Health (9) published a table of low-risk doses of ethanol in various countries (Table

Table 1. Low-risk ethanol doses in various countries (in alphabetical order)

\begin{tabular}{|c|c|c|c|c|}
\hline \multirow[t]{2}{*}{ Country } & \multicolumn{2}{|c|}{$\begin{array}{l}\text { Daily limit of ethanol } \\
\text { (g) }\end{array}$} & \multicolumn{2}{|c|}{$\begin{array}{l}\text { Weekly limit of ethano } \\
\text { (g) }\end{array}$} \\
\hline & Men & Women & Men & Women \\
\hline Albania & 20 & 20 & - & - \\
\hline Australia & 20 & 20 & - & - \\
\hline Austria & 24 & 16 & 168 & 112 \\
\hline $\begin{array}{l}\text { Bosnia and } \\
\text { Herzegovina }\end{array}$ & 20 & 10 & - & - \\
\hline Bulgaria & 16 & 8 & - & - \\
\hline Canada & 40.3 & 26.9 & 201.7 & 134.5 \\
\hline Croatia & 20 & 10 & - & - \\
\hline Czech Republic & 24 & 16 & - & - \\
\hline Denmark & - & - & 168 & 84 \\
\hline Estonia & 20 & 10 & - & - \\
\hline Finland & 20 & 10 & - & - \\
\hline France & 30 & 20 & - & - \\
\hline Georgia & 20 & 20 & - & - \\
\hline Germany & 20 & 10 & - & - \\
\hline Hungary & 2 units* & 1 unit* $^{*}$ & - & - \\
\hline Ireland & - & - & 168 & 112 \\
\hline Italy & $24-36$ & $12-24$ & - & - \\
\hline Macedonia & 28.4 & 14.2 & - & - \\
\hline Malta & - & - & 168 & 112 \\
\hline Netherlands & 20 & 10 & - & - \\
\hline New Zealand & 30 & 20 & 150 & 100 \\
\hline Poland & 40 & 20 & 280 & 140 \\
\hline Portugal & 24 & 16 & - & - \\
\hline Serbia & 26 & 13 & - & - \\
\hline Slovenia & 20 & 10 & 140 & 70 \\
\hline Spain & - & - & - & - \\
\hline Catalonia & 40 & 20 & 280 & 170 \\
\hline Sweden & 24 & 15 & 168 & 108 \\
\hline United Kingdom & 24-32 & $16-24$ & - & - \\
\hline USA & $14-28$ & 14 & 196 & 98 \\
\hline
\end{tabular}

Source: National Institute of Public Health, Czech Republic

*unit: $200 \mathrm{ml}$ of beer or $100-150 \mathrm{ml}$ of wine or $20-30 \mathrm{ml}$ of spirits 
Table 2. Alcohol content and calorie values of the most common alcoholic beverages

\begin{tabular}{|l|c|c|c|}
\hline Beverage & Quantity (I) & Alcohol content (g) & kcal/kJ \\
\hline Beer $10^{\circ}$ & 0.5 & 16 & $160 / 670$ \\
\hline Beer $12^{\circ}$ & 0.5 & 18 & $170 / 720$ \\
\hline White wine & 0.2 & 15 & $108 / 450$ \\
\hline Red wine & 0.2 & 19 & $134 / 560$ \\
\hline Sparkling wine & 0.1 & 10 & $80 / 334$ \\
\hline Fortified wine* & 0.1 & 15 & $99-214 / 414-586^{* *}$ \\
\hline Spirits & 0.05 & 20 & $140 / 586$ \\
\hline
\end{tabular}

*Port wine, sherry, vermouth; **Depending on sugar content

1). "Low-risk" alcohol consumption recommended for the Czech Republic should not exceed $16 \mathrm{~g}$ of $100 \%$ ethanol/day for women and $24 \mathrm{~g} /$ day for men; at least two days a week should be alcohol free, and the dose of ethanol during binge drinking should not exceed $40 \mathrm{~g}$. In practice, this means one standard drink daily for five days at most and two standard drinks at most when binge drinking. Table 2 shows the alcohol content and calorie values of the most common alcoholic beverages in the Czech Republic.

As recommended in the joint European Guidelines on CVD prevention (10), daily consumption of alcoholic drinks should not exceed $20 \mathrm{~g}$ of ethanol in men and $10 \mathrm{~g}$ of ethanol in women. These amounts should be considered the highest acceptable limits, but alcohol consumption in general should be discouraged. In the light of the most recent data, this approach seems to be a reasonable compromise.

\section{Conflict of Interests}

None declared

\section{REFERENCES}

1. Ronksley PE, Brien SE, Turner BJ, Mukamal KJ, Ghali WA. Association of alcohol consumption with selected cardiovascular disease outcomes: a systematic review and meta-analysis. BMJ. 2011;342:d671. doi: 10.1136/ bmj.d671.

2. Rehm J, Baliunas D, Borges GL, Graham K, Irving H, Kehoe T, et al. The relation between different dimensions of alcohol consumption and burden of disease: an overview. Addiction. 2010;105(5):817-43.
3. Shaper AG, Wannamethee G, Walker M. Alcohol and mortality in British men: explaining the U-shaped curve. Lancet. 1988;2(8623):1267-73.

4. Brien SE, Ronksley PE, Turner BJ, Mukamal KJ, Ghali WA. Effect of alcohol consumption on biological markers associated with risk of coronary heart disease: systematic review and meta-analysis of interventional studies. BMJ. 2011 Feb 22;342:d636. doi: 10.1136/bmj.d636.

5. Bennett DA, Holmes MV. Mendelian randomisation in cardiovascular research: an introduction for clinicians. Heart. 2017;103(18):1400-7.

6. Edenberg HJ. The genetics of alcohol metabolism: role of alcohol dehydrogenase and aldehyde dehydrogenase variants. Alcohol Res Health. 2007;30(1):5-13.

7. Holmes MV, Dale CE, Zuccolo L, Silverwood RJ, Guo Y, Ye Z, et al. Association between alcohol and cardiovascular disease: Mendelian randomisation analysis based on individual participant data. BMJ. 2014;349:g4164. doi: 10.1136/bmj.g4164.

8. Wood AM, Kaptoge S, Butterworth AS, Willeit P, Warnakula S, Bolton $\mathrm{T}$, et al. Risk thresholds for alcohol consumption: combined analysis of individual-participant data for 599912 current drinkers in 83 prospective studies. Lancet. 2018;391(10129):1513-23.

9. Ruprich J, editor. An overview of national guidances for moderate consumption of alcoholic beverages: low-risk ethanol doses. Brno: NIPH 2015.

10. Piepoli MF, Hoes AW, Agewall S, Albus C, Brotons C, Catapano AL, et al. European Guidelines on cardiovascular disease prevention in clinical practice: The Sixth Joint Task Force of the European Society of Cardiology and Other Societies on Cardiovascular Disease Prevention in Clinical Practice (constituted by representatives of 10 societies and by invited experts) Developed with the special contribution of the European Association for Cardiovascular Prevention \& Rehabilitation (EACPR). Eur Heart J. 2016;37(29):2315-81. 